\title{
Blockade of vascular endothelial growth factor-A/receptor 2 exhibits a protective effect on angiotensin-II stimulated podocytes
}

\author{
XIANGCHUN LIU*, HONG ZHANG ${ }^{*}$, QUN WANG, KEZHOU YU, RONG WANG and JING SUN \\ Department of Nephrology, Shandong Provincial Hospital, Shandong University, Jinan, Shandong 250021, P.R. China
}

Received May 19, 2014; Accepted April 10, 2015

DOI: $10.3892 / \mathrm{mmr} .2015 .3911$

\begin{abstract}
Vascular endothelial growth factor (VEGF) and Angiotensin II (Ang-II) are important in glomerulosclerosis, which is one of the main causes of chronic kidney disease. Previous studies have demonstrated that angiotensin type 1 receptor blocker (ARB) can inhibit the synthesis of VEGF mediated by Ang-II and can effectively treat diabetic nephropathy. In the present study, the expression of VEGF and its receptors (VEGFR1/VEGFR2) was examined in Ang-II stimulated podocytes, which were treated with SU5416, a specific VEGFR2 inhibitor. The protein expression of synaptopodin, VEGFR1/2, phosphorylated VEGFR2 and extracellular signal-regulated kinases (ERK) was assessed by western blot analysis. The mRNA expression of transforming growth factor (TGF)- $\beta 1$ was examined by reverse transcription-quantitative polymerase chain reaction. It was observed that Ang-II increased the expression of VEGF-A and VEGFR2. Simultaneously, the increased expression of phosphorylated (p-)VEGFR2 and p-ERK induced by Ang-II was downregulated by SU5416. SU5416 can decrease the expression of synaptopodin and increase the expression of TGF- $\beta 1$ induced by Ang-II as well as ARB treatment. The expression of VEGFR1 remained unchanged by either Ang-II or SU5416 treatment. However, the normal podocytes administered SU5416 alone showed low levels of synaptopodin and high expression of TGF- $\beta 1$ compared with the control. In conclusion, VEGF-A/VEGFR2 may be essential for podocytes in a normal state. It is suggested that blockade of VEGF-A/VEGFR2 may exhibit a protective effect on Ang-II stimulated podocytes.
\end{abstract}

Correspondence to: Dr Jing Sun, Department of Nephrology, Shandong Provincial Hospital, Shandong University, 324 Jingwu Weiqi Road, Jinan, Shandong 250021, P.R. China

E-mail: jingsuncn@126.com

*Contributed equally

Key words: podocytes, vascular endothelial growth factor receptor 2, angiotensin-II

\section{Introduction}

Chronic kidney disease (CKD) often begins with urinary protein loss (proteinuria), an early sign of kidney injury that constitutes a risk factor for further progressive destruction of the kidney. Proteinuria stems from injury to podocytes. Loss of podocytes or failure of podocyte function contributes to the development of glomerulosclerosis, which is the final stage of various renal diseases (1). A study demonstrated that there may be a close association between vascular endothelial growth factor (VEGF) and proteinuria (2). VEGF is an important regulator of angiogenesis. In addition, podocytes are the major source of VEGF production in the glomerulus $(3,4)$. The VEGF family incorporates five ligands that can bind differentially to three receptor tyrosine kinases (VEGFR-1, -2 and -3). Recent studies have demonstrated that plasma VEGF levels are increased in CKD and podocyte-derived VEGF is upregulated in the early stages of diabetic nephropathy (5-7). The therapeutic effects of anti-VEGF strategies were partially shown to prevent albuminuria in diabetic rodents and prevent the complications of CKD (8-11). It was reported that primary or secondary elevations in VEGFR-1 (sFlt-1) may lead to decreased tissue levels of VEGF-A and the organ defects observed in preeclampsia (12). By contrast, Eremina et al (13) demonstrated that VEGF production by podocytes is also required to maintain the integrity of the glomerular basement membrane once fully formed, as pharmacologic and genetic inhibition of VEGF in mature podocytes resulted in glomerular endothelial cell damage and thrombotic microangiopathy (13).

From existing literature, the role of VEGF production by podocytes is well established; however, the expression and/or function(s) of VEGF receptors within the podocyte is less clear and is an area of discussion. It is also unclear whether VEGF can directly affect podocytes or is central to the pathogenesis of proteinuria. The aim of the present study was to identify the role of VEGF and its receptors in podocytes.

\section{Materials and methods}

Antibodies and reagents. The following commercially available antibodies were used: Anti- $\beta$-actin (cat. no. sc-8432), anti-synaptopodin (cat. no. sc-50459), anti-VEGFR1 (cat. no. sc-271789), anti-phosphorylated-VEGFR2 (cat. no. sc-16629), anti-VEGFR2 (cat. no. sc-6251) and 
horseradish peroxidase-conjugated rabbit anti-mouse immunoglobulin G secondary antibody (cat. no. sc-358918; Santa Cruz Biotechnology Inc., Santa Cruz, CA, USA), horseradish peroxidase-conjugated anti-rabbit secondary antibody (cat. no. sc-2007; Santa Cruz Biotechnology, Inc.) anti-phosphorylated-extracellular signal-regulated kinases (ERK; Cell Signaling Technology Inc., Beverly, MA, USA). SU5416 an inhibitor of VEGFR2 was obtained from Pfizer Inc. (New York, NY, USA). A bicinchoninic acid (BCA) protein assay kit and nitrocellulose membranes were obtained from Bio-Rad (Hercules, CA, USA). ELISA for the Quantikine mouse VEGF-A immunoassay was purchased from R\&D Systems (Minneapolis, MN, USA). A RNAeasy Mini kit was obtained from Qiagen (Hilden, Germany).

Cell cultures. A thermosensitive, SV40-transfected immortalized mouse podocyte cell line was provided by Dr Peter Mundel (Mount Sinai School of Medicine, New York, NY, USA). The conditionally immortalized mouse podocytes carry a temperature-sensitive variant of the SV-40 large T antigen (tsA58) that is stimulated by mouse interferon (IFN) $-\gamma$ and is stable at $33^{\circ} \mathrm{C}$ as described previously (14). At $33^{\circ} \mathrm{C}$, cells were left to proliferate in RPMI-1640 medium (Sigma-Aldrich, St. Louis, MO, USA) supplemented with 10-20 U/ml mouse recombinant IFN- $\gamma$ (Peprotech, Rocky Hill, NJ, USA) 100 U/ml penicillin/streptomycin (Sigma-Aldrich) and 10\% fetal calf serum (FCS; Fuzhou Maixin Biotechnology Development Co., Ltd., Fuzhou, China). To induce differentiation, cells were thermoshifted to $37^{\circ} \mathrm{C}$ for two weeks without IFN- $\gamma$. These cells showed an epithelial morphology with a polyhedral shape and were detected by synaptopodin, a differentiated podocyte-specific marker, using immunofluorescence staining (Fig. 1A) (15).

Experimental design. Stimulation experiments were performed with Angiotensin II (Ang-II; Fuzhou Maixin Biotechnology Development Co., Ltd). Ang-II concentrations $\left(10^{-5}, 10^{-6}, 10^{-7}\right.$, $10^{-8}$ and $10^{-9} \mathrm{M}$ ) resulting in significant VEGF-A activation were used for time course experiments $(24,36,48$ and $60 \mathrm{~h})$. The synchronized differentiated podocytes were either untreated or treated with Ang-II $\left(10^{-7} \mathrm{M}\right)$ for $48 \mathrm{~h}$ to achieve a podocyte injury pattern. To define the effect of the angiotensin type 1 receptor blocker (ARB), irbesartan $\left(10^{-4} \mathrm{M}\right.$; Sigma-Aldrich), or a VEGF receptor 2 inhibitor, SU5416, on Ang-II-stimulated podocytes $(16,17)$, these two compounds were added to podocytes $1 \mathrm{~h}$ prior to treatment with Ang-II. All experimental groups were collected for extraction of total RNA and protein after exposure to experimental conditions for $48 \mathrm{~h}$. Three independent experiments were performed.

ELISA. VEGF-A concentrations were measured in the supernatant of podocytes by ELISA using a Quantikine human VEGF-A Immunoassay. The supernatant was left on cells for $48 \mathrm{~h}$. All experiments were performed in three independent experimental setups.

Immunofluorescent staining. Differentiated mouse podocytes were seeded on collagen I-coated glass coverslips (Shanghai Precision \& Scientific Instrument Co., Ltd., Shanghai, China) for two weeks at $37^{\circ} \mathrm{C}$, washed three times in $1 \mathrm{X}$ phosphate-buffered saline (PBS) for $5 \mathrm{~min}$, then fixed with $4 \%$ formaldehyde for $30 \mathrm{~min}$ at $37^{\circ} \mathrm{C}$ and rinsed with PBS. Subsequently, 0.2\% Triton X-100 (Sigma-Aldrich) in PBS was added for $20 \mathrm{~min}$ to permeabilize the cells. Podocytes on glass coverslips were washed with PBS again, blocked with $2 \% \mathrm{FCS}$ for $30 \mathrm{~min}$ at $37^{\circ} \mathrm{C}$, then incubated with an anti-mouse synaptopodin antibody in 1:200 dilution at $4^{\circ} \mathrm{C}$ overnight. Cells were then washed with PBS and incubated with secondary antibody in the dark for $30 \mathrm{~min}$ at room temperature. Photomicrographs of each section of the groups were observed at x100 magnification with a fluorescence microscope (BX61; Olympus Corporation, Tokyo, Japan).

Western blot analysis. Podocytes from five experimental groups (control, Ang-II, SU5416, Ang-II + SU5416, and Ang-II + irbesartan) were collected and total protein concentration was measured using a BCA protein assay kit. Protein $(30 \mu \mathrm{g})$ was electrophoresed on 5\% SDS-PAGE and transferred onto a nitrocellulose membrane. The membrane was blocked in 5\% non-fat milk in Tris-buffered saline/0.15\% Tween-20 (TBST) for $2 \mathrm{~h}$ at room temperature and hybridized overnight at $4{ }^{\circ} \mathrm{C}$ with one of the primary antibodies. After three washes in TBST, membranes were incubated with the secondary antibody for $1 \mathrm{~h}$ at room temperature. The specific protein band on membranes was detected by computer-assisted video densitometry and its density was measured by using the Image Reader LAS-4000 (Fujifilm Corp., Tokyo, Japan).

RNA isolation and reverse transcription-quantitative polymerase chain reaction ( $R T-q P C R)$. Total mRNA was purified from differentiated mouse podocytes using an RNAeasy Mini kit (Qiagen, Hilden, Germany) according to the manufacturer's instructions. RNA concentration was assessed by spectrophotometry at 260 and $280 \mathrm{~nm}$. Total mRNA $(1 \mu \mathrm{g})$ was used for reverse transcription to generate cDNA following a reported protocol (18). The reverse transcription kits were provised by Bioteke Corp. (Beijing, China). The resulting cDNA was used as a template for PCR amplification. Next, cDNA was amplified with the following PCR specific primers: Sense: 5'-GCC TGG TCT ACA TAC AGA GTG AG-3' and antisense: 5'-TCT AGT CCT CAG ACC CAG TCA TA-3' for mouse transforming growth factor (TGF)- $\beta 1$; and sense: 5 '-GTC CCT CAC CCT CCC AAA AG-3' and antisense: 5'-GCT GCC TCA ACA CCT CAA CCC-3' for mouse $\beta$-actin. PCR conditions were as follows: Denaturation at $92^{\circ} \mathrm{C}$ for $2 \mathrm{~min}, 35$ cycles of denaturation at $94^{\circ} \mathrm{C}$ for $30 \mathrm{sec}$, annealing at $55^{\circ} \mathrm{C}$ for $30 \mathrm{sec}$, and extension at $72^{\circ} \mathrm{C}$ for $30 \mathrm{sec}$, followed by final extension at $72^{\circ} \mathrm{C}$ for $10 \mathrm{~min}$. The RT-qPCR products were run on a $2 \%$ agarose gel with ethidium bromide staining by electrophoresis. The predicted band sizes were 699 bp for TGF- $\beta 1$ and 266 bp for mouse $\beta$-actin. The abundance of TGF- $\beta 1$ mRNA was normalized to $\beta$-actin.

Statistical analysis. All data are presented as the mean \pm standard error of the mean. Differences between groups were analyzed by a two-sample t-test or one-way analysis of variance using GraphPad Prism software version 3.03 (GraphPad Software, San Diego, CA, USA). P<0.05 was considered to indicate a statistically significant difference. 
A

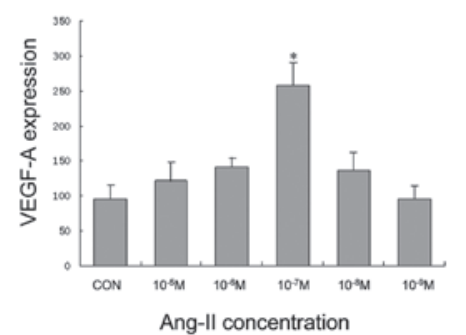

B

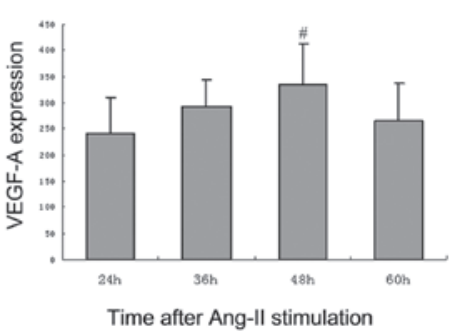

Figure 1. Activation of VEGF-A in differentiated mouse podocytes after stimulation with Ang-II. (A) Dose-response for Ang-II in podocytes treated with $10^{-5}$, $10^{-6}, 10^{-7}, 10^{-8}$ and $10^{-9} \mathrm{M}$ Ang-II. The strongest effect on VEGF-A was detected for $10^{-7} \mathrm{M}$ Ang-II. ${ }^{*} \mathrm{P}<0.001$, compared with the other groups. (B) Time course for $10^{-6}, 10^{-7}$ and $10^{-8} \mathrm{M}$ Ang-II treatment in podocytes. VEGF-A levels reached a peak $48 \mathrm{~h}$ after Ang-II stimulation. ${ }^{*} \mathrm{P}<0.05$, compared with the other groups. VEGF-A, vascular endothelial growth factor-A; Ang-II, angiotensin II.

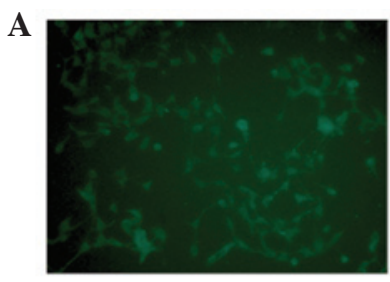

D

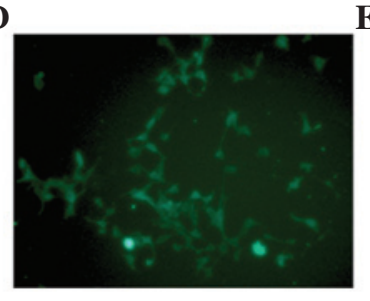

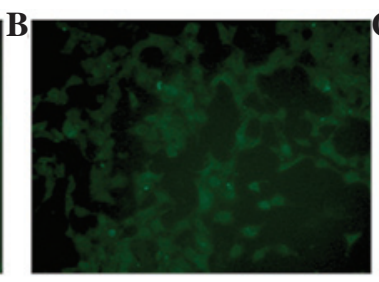

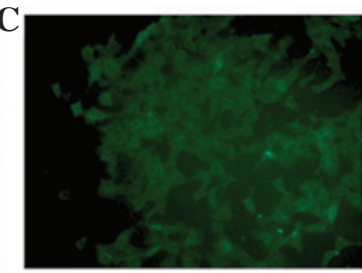

E

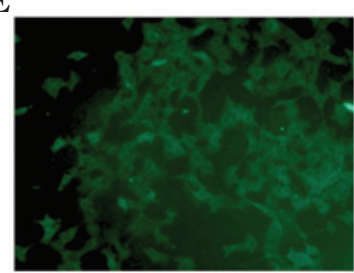

Figure 2. Immunofluorescent staining of synaptopodin presented green. (A) Immortalized mouse podocyte cells expressed synaptopodin in the normal control group. (B) The staining of synaptopodin decreased with the stimulation of angiotensin II (10 $\left.{ }^{-7} \mathrm{M}\right)$ for $48 \mathrm{~h}$. (C) SU5416 showed to decrease the expression of synaptopodin compared with control. (D) The decrease in synaptopodin was recovered by the treatment with SU5416. (E) In addition, irbesartan restored the expression of synaptopodin partially. Magnification, x100.

\section{Results}

Ang-II induces the expression of VEGF-A in podocytes. The integrity of VEGF-A was demonstrated by stimulation of Ang-II in podocytes. Concentrations of $10^{-5}, 10^{-6}, 10^{-7}, 10^{-8}$ and $10^{-9} \mathrm{M}$ Ang-II were used for the dose response experiment. Treatment with $10^{-7} \mathrm{M}$ Ang-II led to a strong activation of VEGF-A (increased 2.7-fold, $\mathrm{P}<0.001$, in three independent experiments) (Fig. 1A). In addition, time course experiments were performed to examine the time dependency of VEGF-A activation. Based on the dose-response experiments, $10^{-6}, 10^{-7}$, and $10^{-8} \mathrm{M}$ Ang-II were used for the time course experiments. VEGF-A peaked $48 \mathrm{~h}$ following stimulation with Ang-II (Fig. 1B). Thus, in the following experiments, a treatment dose of $10^{-7} \mathrm{M}$ Ang-II for $48 \mathrm{~h}$ was used.

SU5416 is observed to ameliorate the decrease in the protein level of synaptopodin in Ang-II-injured podocytes. Following treatment with Ang-II $\left(10^{-7} \mathrm{M}\right)$ for $48 \mathrm{~h}$, the synchronized differentiated podocytes were injured. The protein level of synaptopodin in injured podocytes was significantly reduced $(0.41 \pm 0.101$ vs. $0.12 \pm 0.041, P=0.003$ ) (Figs. 2 and 3). It was demonstrated that SU5416 restored the protein level of synaptopodin for blockade of VEGFR2, which was demonstrated by immunofluorescence (Fig. 2) and western blot analysis
A

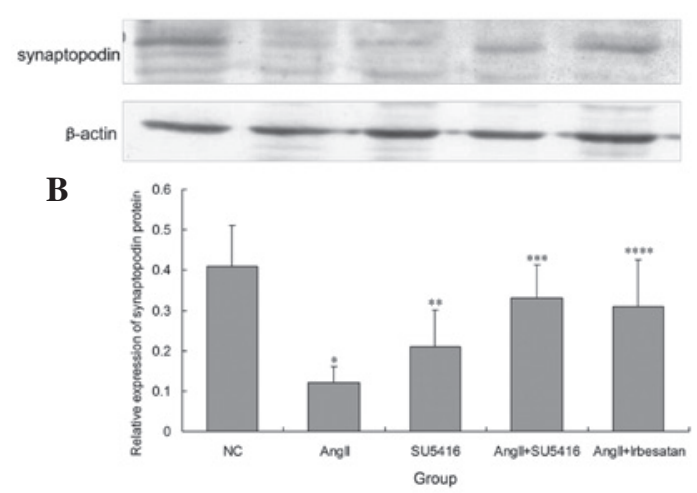

Figure 3. Expression of synaptopodin was analyzed by western blot analysis. (A) Comparison of synaptopodin protein levels between the groups. (B) Relative expression of synaptopodin protein. With Ang-II $\left(10^{-7} \mathrm{M}\right)$ stimulation, the protein level of synaptopodin was significantly reduced $\left({ }^{*} \mathrm{P}=0.003\right.$, compared with the NC group) and restored by SU5416 $\left(^{* * * *} \mathrm{P}=0.002\right.$, compared with the Ang-II group). Irbesartan $\left(10^{-4} \mathrm{M}\right)$ restored the protein level of synaptopodin in AngII-stimulated mouse podocytes ${ }^{* * * *} \mathrm{P}=0.013$, compared with the Ang-II group). In a normal state SU5416 reduced the expression of synaptopodin $\left({ }^{* *} \mathrm{P}=0.008\right.$, compared with the NC group). AngII, angiotensin II.

$(0.12 \pm 0.041$ vs. $0.33 \pm 0.084, \mathrm{P}=0.002$; Fig. 3$)$. Compared with the control, the expression of synaptopodin was decreased in 
A
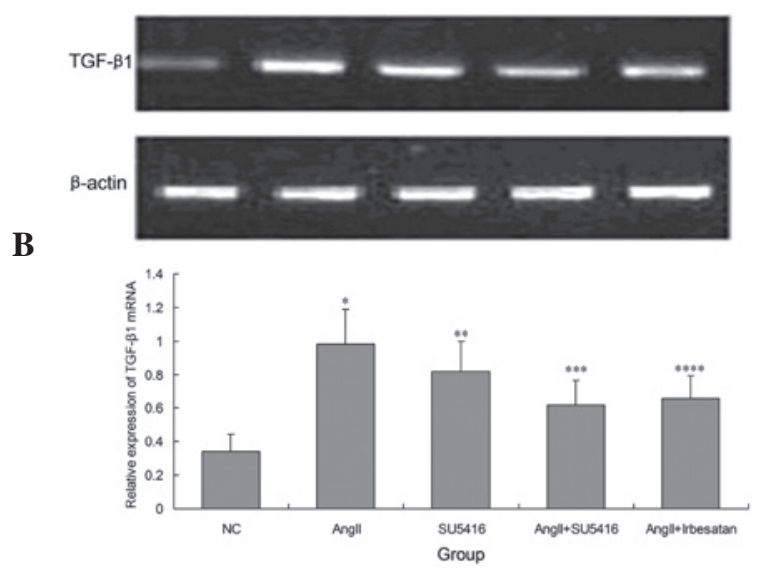

Figure 4. TGF- $\beta 1$ mRNA levels. (A) Comparison of TGF- $\beta 1$ mRNA levels between the groups by reverse transription-quantitative polymerase chain reaction. (B) Relative expression of TGF- $\beta 1$ mRNA. The expression of TGF- $\beta 1$ was significantly increased by Ang-II $\left({ }^{*} \mathrm{P}=0.009\right.$, compared with the NC group). SU5416 was shown to reduce the high level of TGF- $\beta 1$ mRNA ( ${ }^{* * *} \mathrm{P}=0.011$, compared with the Ang-II group), whereas irbesartan reversed the level of TGF- $\beta 1$ mRNA in Ang-II-injured podocytes $\left({ }^{* * * *} \mathrm{P}=0.019\right.$, compared with the Ang-II group). In a normal state, SU5416 alone showed the same effect on TGF- $\beta 1$ as Ang-II ( ${ }^{* *} \mathrm{P}=0.012$, compared with the NC group). AngII, angiotensin II.

\section{$\mathbf{A}$}

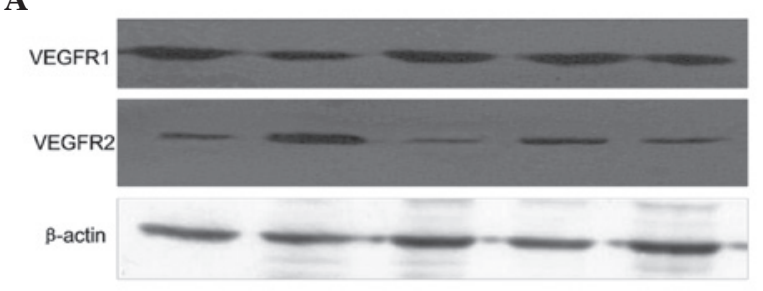

$\mathbf{B}$

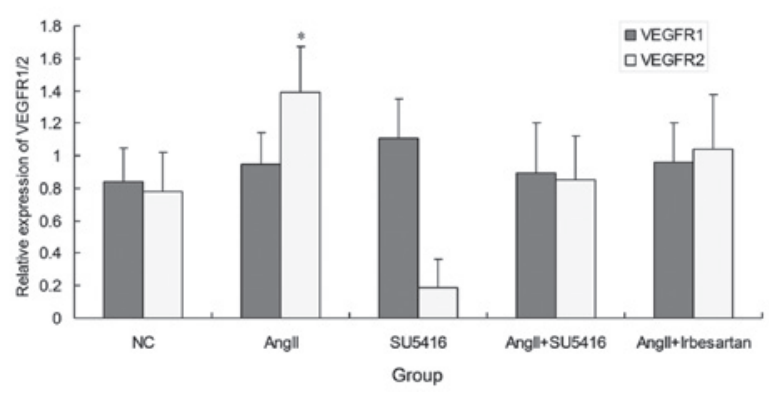

Figure 5. Expression of the two receptors (VEGFR1/VEGFR2) assessed by western blot analysis. (A) Comparison of the protein levels of VEGFR1/VEGFR2 between the groups. (B) Relative expression of VEGFR1/2. Ang-II significantly increased VEGFR2 expression ( $\mathrm{P}=0.024$ compared with NC group). The expression of VEGFR1 remained unchanged following either Ang-II and SU5416 treatment (P>0.05). VEGF-A, vascular endothelial growth factor-A; AngII, angiotensin II.

the normal podocytes treated with SU5416 alone $(0.41 \pm 0.101$ vs. 0.21 $\pm 0.092, \mathrm{P}=0.008$; Fig. 2).

In order to evaluate whether ARBs have beneficial effects on injured podocytes, $10^{-4} \mathrm{M}$ irbesartan was added to Ang-II treated podocytes for $48 \mathrm{~h}$. It was observed that irbesartan also partially restored the protein level of synaptopodin (0.12 \pm 0.041 vs. $0.31 \pm 0.117, \mathrm{P}=0.013$; Figs. 2 and 3$)$.
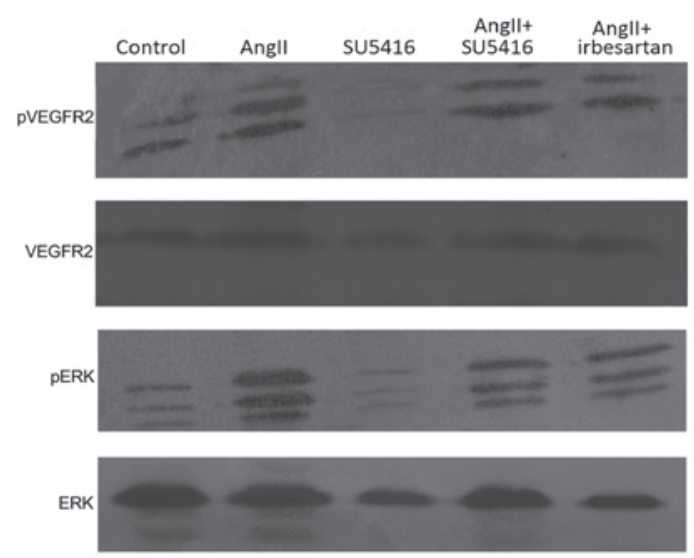

Figure 6. Phosphorylation of VEGFR2 and ERK assessed by western blot analysis. Ang-II could upregulate the phosphorylation of VEGFR2, and significant inhibition of phosphorylated VEGFR2 was observed in podocytes treated with SU5416, the same effect was observed on pERK as well as pVEGFR2. VEGFR2, vascular endothelial growth factor receptor-2; ERK, extracellular signal-regulated kinases; $p$, phosphorylated.

SU5416 decreases TGF- $\beta 1$ mRNA expression in Ang-II-injured podocytes. It was then examined whether blocking the VEGF/VEGFR2 pathway effects TGF- $\beta 1$, a cytokine involved in extracelluar fibrosis. Thus, SU5416 (1 $\mu \mathrm{mol} / \mathrm{l})$ was added to Ang-II injured podocytes for $48 \mathrm{~h}$ in order to analyze TGF- $\beta 1$ mRNA expression. RT-qPCR revealed that the high expression of TGF- $\beta 1$ mRNA in Ang-II-injured podocytes was decreased by SU5416 through the blockade of VEGFR2 $(0.98 \pm 0.211$ vs. $0.62 \pm 0.146$, $\mathrm{P}=0.011$; Fig. 4). Furthermore, it was observed that $10^{-4} \mathrm{M}$ irbesartan reversed the expression of TGF- $\beta 1$ mRNA in injured podocytes $(0.98 \pm 0.211$ vs. $0.65 \pm 0.134, \mathrm{P}=0.009)$. In the normal state, treatment with SU5416 alone was observed to increase the mRNA expression of TGF- $\beta 1(0.34 \pm 0.104 \mathrm{vs}$. $0.82 \pm 0.178, \mathrm{P}=0.012$; Fig. 4).

Ang-II exhibits no effect on VEGFRl and enhances the expression of $V E G F R 2$. In addition to the expression of synaptodin and TGF- $\beta 1$, the expression of two major VEGF-A receptors VEGFR1 (flt-1) and VEGFR2 (flk-1) were investigated following treatment with Ang-II and SU5416. The VEGFR2 (flk-1) level was enhanced markedly by Ang-II stimulation compared with control as observed using western blot analysis $(1.39 \pm 0.327$ vs. $0.78 \pm 0.246, \mathrm{P}=0.024)$. Вy contrast, VEGFR1 (flt-1) levels were not affected by treatment with Ang-II and SU5416 (P>0.05; Fig. 5)

SU5416 downregulates the phosphorylation of VEGFR2 and ERK. Based on the results that demonstrated that the expression of VEGFR2 was enhanced by Ang-II, the level of phosphorylated VEGFR2 and its downstream factor, ERK, were examined. It was observed that the expression of phosphorylated VEGFR2 was significantly increased by Ang-II, and was decreased by SU5416. Compared with control, phosphorylation of ERK was also upregulated by treatment with Ang-II. Blockade of VEGFR2 decreased the level of phosphorylated-ERK (Fig. 6).

Irbesartan showed the same effect on the phosphorylation of VEGFR2 and ERK as SU5416 (Fig. 6). 


\section{Discussion}

Recently, studies have shown that modulating angiogenesis-related factors has potential therapeutic effects in CKD (19-21). In the present study, the increased expression of VEGF-A was observed in cultured podocytes stimulated with Ang-II. Ang-II was observed to increase the expression of VEGFR2 (flk-1) and its level of phosphorylation; however, it had a lower effect on VEGFR1 (flt-1). Blockade of VEGFR2 by SU5416 downregulated the phosphorylation of VEGFR2 and ERK. Simultaneously, it recovered the expression of synaptopodin and decreased the level of TGF $\beta 1$ in Ang-II-induced podocytes. It was also demonstrated that the normal podocytes were injured by treatment with SU5416 alone. Irbesartan showed the same effect on the Ang-II stimulated podocytes as SU5416.

VEGF-A is required for the development of the glomerular filtration barrier (22). Although a previous study (22) established a link between increased VEGF-A, and glomerular injury and proteinuria, it remains unknown whether increased VEGF-A is causative or simply a consequence of these pathologic processes. There remains controversy regarding the beneficial and deleterious effects of VEGF-A on the kidney as certain studies suggested that VEGF may protect against renal injury $(7,8)$. In the present study, it was observed that blockade of VEGF-A/VEGFR2 may injure podocytes, suggesting that VEGF-A/VEGFR2 is essential for the normal growth of podocytes. When the expression of VEGF-A/VEGFR2 was lower than the normal level, the podocytes were damaged. This is consistent with the findings of Eremina et al (13).

Simultaneously increased VEGF-A expression was observed in Ang-II-stimulated podocytes. Blockade of VEGFR2 was shown to reduce the phosphorylation of VEGFR2 and ERK, and ammeliorate the change in expression of synaptopodin and TGF- $\beta 1$. Thus, VEGF A autocrined by podocytes treated with AngII may have an important effect on the extracellular matrix (ECM) and cell morphology through VEGFR2. Simultaneously, it may be postulated that the proportion balance of phosphorylated VEGFR2 has been broken when the podocytes is injured by Ang-II. Hohenstein et al (23) found that the majority of cells at sites of prominent injury, such as crescents, demonstrated high expression levels of VEGFR1 in a large number of renal biopsies from patients with glomerulonephritis (GN). However, numerous glomeruli had less intense VEGFR1 expression, despite obvious morphological changes such as increased cellularity or matrix. By contrast podocytic VEGFR2 expression was more prominent in biopsies with GN. The results of the present study showed that VEGFR1 had not been influenced by Ang-II and suggested that blockade of VEGFR2 had a beneficial effect on Ang-II-stimulated podocytes. It could be deduced that the roles of VEGR1/2 were different in podocytes. While VEGFR1 may be important in cell proliferation and survival, VEGFR2 appeared to be involved in the podocytes architecture and morphology. When phosphorylation of VEGFR2 was impaired, cytokines involved in morphology and matrix, such as synaptopodin and TGF- $\beta 1$ exhibited altered levels of expression. These data reveal that VEGF-A activation of VEGFR2, not VEGFR1, directly resulted in the damage of podocyte ECM and morphology by Ang-II.

To identify the mechanisms by which VEGF-A activated of VEGFR2 in stimulated Ang-II-injured podocytes, VEGF-A-induced signaling pathways were analyzed by blockade of VEGF-A/VEGFR2 using SU5416. It was shown that VEGF-A/VEGFR2 activation in Ang-II-injured podocytes stimulated phosphorylation of ERK. To the best of our knowledge, phosphorylation of ERK is the upstream regulator of TGF $\beta 1$. Thus, this suggested that the phosphorylation of ERK was required for the VEGF-A/VEGFR2-induced damage of podocytes treated with Ang-II.

Synaptopodin, an actin-associated protein, is expressed only in completely differentiated podocytes and in the telencephalic synapses (24). Podocytes are important in the maintenance of renal glomerular function and in the pathogenesis of glomerulosclerosis (25). It is postulated that synaptopodin modulates the actin-based contractile microfilament apparatus of the podocyte foot processes and the integrity of matured podocytes. Synaptopodin may be involved in the development of proteinuric renal diseases and maintenance of the glomerular filtration barrier $(26,27)$. The increasing level of synaptopodin in podocytes has been revealed with proteinuria (28). The present study examined that blockade of VEGF-A/VEGFR2 by the VEGFR2 inhibitor ameliorated the decrease in the protein level of synaptopodin in Ang-II-injured podocytes. This result is in line with anti-VEGF-related proteinuria in non-diabetic CKD (29).

Although it is now evident that damage is transmitted from podocyte to podocyte, and from podocyte to other glomerular cells, it remains largely unknown how these transmissions occur, and what factors are involved (30). Possible mechanisms include increased toxic substance(s) secreted in an autocrine or paracrine manner, such as basic fibroblast grow th factor, TGF- $\beta$, Ang-II and macrophage migration inhibitory factor, and decreased levels of supportive substances for survival of the podocytes. In the present study it was observed that inhibition of VEGF-A/VEGFR2 increased the expression of TGF- $\beta 1$ mRNA in Ang-II-injured podocytes, indicating that blockade of the VEGF pathway may delay the progression of glomerulosclerosis. The observation that blockade of VEGF-A/VEGFR2 was associated with decreased expression of TGF- $\beta 1$ mRNA suggested that podocyte damage may induce further podocyte damage in a positive feedback mechanism, which drives local spread of glomerulosclerosis.

Renin-angiotensin system (RAS) inhibitors have been shown to reduce glomerular permeability and proteinuria. Local elevations in Ang-II signaling mediated by angiotensin type I receptors led to elevations in VEGF expression in endothelial cells in rats (31). Several studies have demonstrated a close correlation between RAS and the level of VEGF in vitro, showing that Ang-II can induce VEGF expression (32-34). In the present study, the therapeutic effects of VEGFR2 inhibition on TGF- $\beta 1$ and synaptopodin were observed compared with ARB in cultured podocytes. It was shown that pretreatment with irbesartan lowered the expression of TGF- $\beta 1$, which is a major mediator of the hypertrophic and prosclerotic changes in the kidney. Furthermore, the results 
suggested another anti-albuminuric molecular mechanism of irbesartan, which restored the levels of synaptopodin in Ang-II-injured podocytes.

In conclusion, the pathway of VEGF-A/VEGFR2 is essential for podocytes in the physiological state. The expression of VEGF-A and VEGFR2 was increased in Ang-II-injured podocytes. The VEGFR2 inhibitor appeared to restore the level of synaptopodin and decrease the expression of TGF- $\beta 1$. It suggested that blockade of VEGF-A/VEGFR2 exhibits beneficial effects on the extracellular matrix and cell morphology in Ang-II-stimulated podocytes.

\section{References}

1. Ma J, Rossini M, Yang HC, Zuo Y, Fogo AB and Ichikawa I Effects of podocyte injury on glomerular development. Pediatr Res 62: 417-421, 2007.

2. Kim NH, Oh JH, Seo JA, et al: Vascular endothelial growth factor (VEGF) and soluble VEGF receptor FLT-1 in diabetic nephropathy. Kidney Int 67: 167-177, 2005.

3. Bates DO and Curry FE: Vascular endothelial growth factor increases hydraulic conductivity of isolated perfused microvessels. Am J Physiol 271: H2520-H2528, 1996.

4. Siemann DW, Brazelle WD and Jürgensmeier JM: The vascular endothelial growth factor receptor-2 tyrosine kinase inhibitor cediranib (Recentin; AZD2171) inhibits endothelial cell function and growth of human renal tumor xenografts. Int J Radiat Oncol Biol Phys 73: 897-903, 2009.

5. Maeshima Y and Makino H: Angiogenesis and chronic kidney disease. Fibrogenesis Tissue Repair 3: 13-30, 2010.

6 . Foster RR, Hole R, Anderson K, et al: Functional evidence that vascular endothelial growth factor may act as an autocrine factor on human podocytes. Am J Physiol Renal Physiol 284 F1263-F1273, 2003.

7. Baharivand N, Zarghami N, Panahi F, et al: Relationship between vitreous and serum vascular endothelial growth factor levels, control of diabetes and microalbuminuria in proliferative diabetic retinopathy. Clin Ophthalmol 6: 185-191, 2012.

8. Ho C, Hsu YC, Tseng CC, et al: Simvastatin alleviates diabetes-induced VEGF-mediated nephropathy via the modulation of Ras signaling pathway. Ren Fail 30: 557-565, 2008.

9. Doi K, Leelahavanichkul A, Hu X, et al: Pre-existing renal disease promotes sepsis-induced acute kidney injury and worsens outcome. Kidney Int 74: 1017-1025, 2008

10. Celletti FL, Waugh JM, Amabile PG, et al: Vascular endothelial growth factor enhances atherosclerotic plaque progression. Nat Med 7: 425-429, 2001.

11. Ferrara N: Role of vascular endothelial growth factor in physiologic and pathologic angiogenesis: therapeutic implications. Semin Oncol 29 (Suppl 16): 10-14, 2002.

12. Sugimoto H, Hamano Y, Charytan D, et al: Neutralization of circulating vascular endothelial growth factor (VEGF) by anti-VEGF antibodies and soluble VEGF receptor 1 (sFlt-1) induces proteinuria. J Biol Chem 278: 12605-12608, 2003.

13. Eremina V, Jefferson JA, Kowalewska J, et al: VEGF inhibition and renal thrombotic microangiopathy. N Engl J Med 358: 1129-1136, 2008.

14. Shankland SJ, Pippin JW, Reiser J and Mundel P: Podocytes in culture: past, present, and future. Kidney Int 72: 26-36, 2007.

15. Krtil J, Pláteník J, Kazderová M, et al: Culture methods of glomerular podocytes. Kidney Blood Press Res 30: 162-174, 2007.
16. Liang XB, Ma LJ, Naito T, et al: Angiotensin type 1 receptor blocker restores podocyte potential to promote glomerular endothelial cell growth. J Am Soc Nephrol 17: 1886-1895, 2006.

17. Mezrich JD, Nguyen LP, Kennedy G, et al: SU5416, a VEGF receptor inhibitor and ligand of the AHR, represents a new alternative for immunomodulation. PLoS ONE 7: e44547, 2012.

18. Westenskow PD, Kurihara T, Aguilar E, et al: Ras pathway inhibtion prevents neovascularization by repressing endothelial cell sprouting. J Clin Invest 123: 4900-4908, 2013.

19. Veron D, Bertuccio CA, Marlier A, et al: Podocyte vascular endothelial growth factor $\left(\operatorname{Vegf}_{164}\right)$ overexpression causes severe nodular glomerulosclerosis in a mouse model of type 1 diabetes. Diabetologia 54: 1227-1241, 2011.

20. Nasu T, Maeshima Y, Kinomura M, et al: Vasohibin-1, a negative feedback regulator of angiogenesis, ameliorates renal alterations in a mouse model of diabetic nephropathy. Diabetes 58: 2365-2375, 2009.

21. Kim W, Moon SO, Lee SY, et al: COMP-angiopoietin-1 ameliorates renal fibrosis in a unilateral ureteral obstruction model. J Am Soc Nephrol 17: 2474-2483, 2006.

22. Eremina V and Quaggin SE: The role of VEGF-A in glomerular development and function. Curr Opin Nephrol Hypertens 13: 9-15, 2004

23. Hohenstein B, Colin M, Foellmer C, et al: Autocrine VEGF-R loop on podocytes during glomerulonephritis in humans. Nephrol Dial Transplant 25: 3170-3180, 2010.

24. Yanagida-Asanuma E1, Asanuma K, Kim K, et al: Synaptopodin protects against proteinuria by disrupting Cdc42:IRSp53: Mena signaling complexes in kidney podocytes. Am J Pathol 171: 415-427, 2007.

25. de Zoysa JR and Topham PS: Podocyte biology in human disease. Nephrology (Carlton) 10: 362-367, 2005.

26. Asanuma K, Kim K, Oh J, et al: Synaptopodin regulates the actin-bundling activity of alpha-actinin in an isoform-specific manner. J Clin Invest 115: 1188-1198, 2005.

27. Faul C, Asanuma K, Yanagida-Asanuma E and Kim K and Mundel P: Actin up: regulation of podocyte structure and function by components of the actin cytoskeleton. Trends Cell Biol 17: 428-437, 2007.

28. Faul C, Donnelly M, Merscher-Gomez S, et al: The actin cytoskeleton of kidney podocytes is a direct target of the antiproteinuric effect of cyclosporine A. Nat Med 14: 931-938, 2008.

29. Izzedine $\mathrm{H}$, Massard C, Spano JP, et al: VEGF signaling inhibition-induced proteinuria: Mechanisms, significance and management. Eur J Cancer 46: 439-448, 2010.

30. Ichikawa I, Ma J, Motojima M and Matsusaka T: Podocyte damage damages podocytes: autonomous vicious cycle that drives local spread of glomerular sclerosis. Curr Opin Nephrol Hypertens 14: 205-210, 2005.

31. Zhao Q, Egashira K, Inoue S, et al: Vascular endothelial growth factor is necessary in the development of arteriosclerosis by recruiting/activating monocytes in a rat model of long-term inhibition of nitric oxide synthesis. Circulation 105: 1110-1115, 2002.

32. Pan P1, Fu H, Zhang L, et al: Angiotensin II upregulates the expression of placental growth factor in human vascular endothelial cells and smooth muscle cells. BMC Cell Biol 11: 36, 2010.

33. Pupilli C, Lasagni L, Romagnani $\mathrm{P}$, et al: Angiotensin II stimulates the synthesis and secretion of vascular permeability factor/vascular endothelial growth factor in human mesangial cells. J Am Soc Nephrol 10: 245-255, 1999.

34. Kang YS, Park YG, Kim BK, et al: Angiotensin II stimulates the synthesis of vascular endothelial growth factor through the p38 mitogen activated protein kinase pathway in cultured mouse podocytes. J Mol Endocrinol 36: 377-388, 2006. 